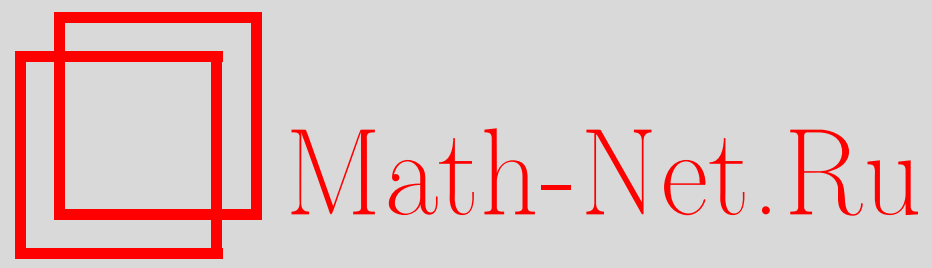

С. А. Кужель, Об элементах нестационарной теории рассеяния для одного класса дифференциально-операторных уравнений, Функи. анализ и его прил., 2000, том 34, выпуск 3, 77-81

DOI: https://doi.org/10.4213/faa317

Использование Общероссийского математического портала MathNet.Ru подразумевает, что вы прочитали и согласны с пользовательским соглашением

http://www. mathnet.ru/rus/agreement

Параметры загрузки:

IP : 44.207 .124 .84

26 апреля 2023 г., 11:39:14

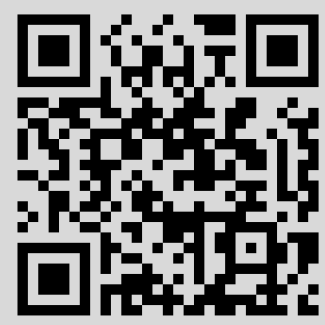


Рассмотрим невырожденную проекцию ориентированного узла на плоскость. Пронумеруем $n$ двойных точек проекции и $n+2$ областей (связные компоненты дополнения). Определим $n \times(n+2)$-матрицу, строки которой отвечают вершинам, а столбцы - областям. Элемент этой матрицы, отвечающий паре вершина-область, не равен нулю, только если область примыкает к вершине. Назовем этот элемент весом вершины в области и определим его по правилу
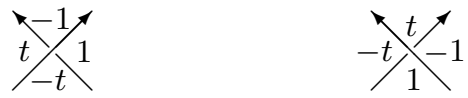

Результат Александера состоит в том, что $n \times n$-минор этой матрицы, полученный вычеркиванием любой пары столбцов, отвечающих соседним областям, с точностью до множителя $\pm t^{m}$ не зависит от вычеркнутой пары столбцов и является инвариантом узла.

Фиксируем пару соседних областей (будем считать, что они имеют номера $n+1$ и $n+2)$ и назовем оставшиеся области допустимыми. Определим «критическую точку функции Морса» как выбор в каждой вершине одной из примыкающих к ней допустимых областей, причем каждая допустимая область должна быть выбрана. Весом «критической точки» назовем произведение весов всех вершин в выбранных областях и знака перестановки, сопоставляющей номеру каждой вершины номер выбранной в этой вершине области. Определим индекс «критической точки» как число вершин, для которых вес выбранной области равен $\pm t$. Тогда из формулы стандартного разложения минора в сумму по перестановкам, очевидно, следует, что каждый коэффициент полинома Александера равен сумме весов всех «критических точек» соответствующего индекса.

\title{
ЛИТЕРАТУРА
}

1. Арнольд В. И., Варченко А. Н., Гусейн-Заде С. М. Особенности дифференцируемых отображений. Т. 2. Наука, М., 1984. 2. Ильюта Г. Г. УМН, 42, вып. 2, 227-228 (1987). 3. Хованский А. Г. Функц. анализ и его прил., 20, вып. 1, 50-61 (1986). 4. A'Campo N. Comm. Math. Helv., 50, No. 2, 233-248 (1975). 5. Alexander J. W. Trans. Amer. Math. Soc., 30, 275-306 (1928). 6. Hibi T. Sugaku Expositions, 8, No. 2, 145-163 (1995). 7. Solomon L. J. Algebra, 168, 521-524 (1994).

Независимый московский университет

Поступило в редакцию 15 декабря 1998 г.

УДК 517.432

\section{Об элементах нестационарной теории рассеяния для одного класса дифференциально-операторных уравнений}

\author{
(C) 2000. С. А. КУжЕЛЬ
}

1. Введение. Принципиальным моментом в развитии нестационарной теории рассеяния для дифференциально-операторного уравнения

$$
u_{t t}=-L u,
$$

\footnotetext{
*Эта работа частично поддержана CRDF, грант UM1-2090.
} 
где $L$ - самосопряженный оператор, действующий в сепарабельном гильбертовом пространстве $\mathfrak{H}$, является определение множества «невозмущенных» операторов $L$, задающих в (1) a priori свободную эволюцию. Сформулируем определение таких операторов в форме, обеспечивающей возможность применения изложенных ниже абстрактных результатов для изучения возмущений в ограниченной области классического волнового уравнения $u_{t t}=\Delta u$.

Пусть $B$ - простой максимальный симметрический оператор в $\mathfrak{H}$. Тогда $B^{2}$ - плотно определенный в $\mathfrak{H}$ симметрический оператор с индексом дефекта $\langle m, m\rangle$, где $m \leqslant \infty$ - ненулевое дефектное число оператора $B$.

ОПРедЕЛЕниЕ. Самосопряженное расширение $L$ оператора $B^{2}$ будем называть невозмущенным, если при всех $u \in D(L)$ справедливо равенство $(L u, u)=$ $\left\|B^{*} u\right\|^{2}$.

Множество всех невозмущенных расширений при фиксированном операторе $B$ обозначим через $\mathfrak{M}_{B}$. Это множество содержит по крайней мере два элемента: расширения Фридрихса $L_{\mu}=B^{*} B$ и Крейна $L_{M}=B B^{*}$. Его полное описание можно найти в $[1,2]$.

Уравнение (1) с произвольным невозмущенным оператором $L$ в правой части будем называть абстрактным волновым уравнением. В [3] указан вид оператора $B$ в пространстве $L_{2}\left(\mathbb{R}^{n}\right)$, для которого свободный Лапласиан $-\Delta$, $D(\Delta)=W_{2}^{2}\left(\mathbb{R}^{n}\right)$, является невозмущенным, а в [1] показано, что с точки зрения подхода Лакса-Филлипса [4] в теории рассеяния абстрактное волновое уравнение и классическое невозмущенное волновое уравнение имеют похожие свойства. Поэтому естественно считать, что абстрактное волновое уравнение определяет свободную эволюцию.

ОпРЕДЕЛЕниЕ. Оператор $\widetilde{L}$, действующий в гильбертовом пространстве $\widetilde{\mathfrak{H}}$, будем называть возмущенныл, если $\widetilde{\mathfrak{H}} \supset \mathfrak{H}$ и $\widetilde{L}$ является положительным самосопряженным расширением оператора $B^{2}$. Возмущенный оператор $\widetilde{L}$ называется 0-возмущенным, если $\widetilde{\mathfrak{H}}=\mathfrak{H}$.

Определим возмущенную эволюцию при помощи уравнения (1) с возмущенным оператором $\widetilde{L}$ в правой части. Выбирая оператор $B$ в различных функциональных пространствах, мы получаем $[1,2]$ конкретные реализации как абстрактного волнового уравнения (парциальное волновое уравнение, волновое уравнение в $\mathbb{R}^{n}$ и т. п.), так и его возмущений (препятствия, потенциалы, неоднородности). Отметим, что в случае волнового уравнения в $\mathbb{R}^{n}$ (нечетное $n>1$ ) примерами такой реализации являются как локализованные в конечной области возмущения волнового уравнения, так и некоторые классы возмущений с неограниченным носителем [1].

Пусть $L-$ произвольный невозмущенный оператор в $\mathfrak{H}$. Пополнение линеала $D(L)$ по норме $\|u\|_{L}^{2}:=(L u, u)$ обозначим через $\mathfrak{H}_{L}$. В энергетическом пространстве $H_{L}=\mathfrak{H}_{L} \oplus \mathfrak{H}$ абстрактное волновое уравнение естественным образом определяет унитарную группу $W_{L}(t)$ решений задачи Коши. Генератор этой группы имеет лебегов спектр $[1,2]$. Аналогично, возмущенное уравнение (1) определяет унитарную (в энергетическом пространстве $\left.H_{\widetilde{L}}=\widetilde{\mathfrak{H}}_{\widetilde{L}} \oplus \widetilde{\mathfrak{H}}\right)$ группу $W_{\widetilde{L}}(t)$ решений задачи Коши.

Следующее утверждение непосредственно вытекает из $[2,3]$. 
ПРЕДЛОЖЕНИЕ 1. Пусть операторь $L$ и $\widetilde{L}$ являются невозмущенньли $u$ возмущенным расширениями оператора $B^{2}$ соответственно. Тогда волновие операторьл $\Omega_{ \pm}=\mathrm{s}_{-} \lim _{t \rightarrow \pm \infty} W_{\widetilde{L}}(-t) W_{L}(t)$ существуют. Eсли оператор $\widetilde{L}$ является 0-возмущенньм, то волновье операторьи $\Omega_{ \pm}$являются полньми.

В [1] построено унитарное отображение $F$ пространства $H_{L}$ на $L_{2}(\mathbb{R}, N)$, которое задает спектральное представление для невозмущенной группы $W_{L}(t)$. Это представление является единственным с точностью до изоморфизма вспомогательного гильбертова пространства $N$, и размерность пространства $N$ равна ненулевому дефектному числу оператора $B$. Оператор рассеяния в спектральном представлении $S=F \Omega_{+}^{*} \Omega_{-} F^{-1}$ можно записать в виде оператора умножения на функцию $S=S(\delta)(\delta \in \mathbb{R})$, значениями которой являются сжимающие операторы в $N$. Функция $S(\delta)$ называется матрицей рассеяния (м.р.) для возмущенной группы $W_{\widetilde{L}}(t)$. Понятно, что м.p. $S(\delta)$ определяется с точностью до изоморфизма пространства $N$. В дальнейшем мы будем отождествлять такие м.р. Следующее утверждение вытекает из [2].

ПРЕДЛОЖЕНИЕ 2. Вид м.p. $S(\delta)$ для возмущенной группь $W_{\widetilde{L}}$ не зависит от въгбора невозмущенного расширения $L \in \mathfrak{M}_{B}$.

Для возмущенной группы $W_{\widetilde{L}}(t)$ можно построить, в терминах оператора $B$, уходящее и приходящее подпространства $[1,2]$. В силу теории Лакса-Филлипса [4] это означает, что м.p. $S(\delta)$ для группы $W_{\widetilde{L}}(t)$ является граничным значением в смысле сильной сходимости аналитической в нижней полуплоскости сжимающей операторнозначной функции $S(z)$. Свойства функции $S(z)$ тесно связаны со свойствами возмущенного оператора $\widetilde{L}$. Целью настоящей заметки является исследование этой связи.

2. Прямая задача. По заданному возмущенному оператору $\widetilde{L}$ построим аналитическое продолжение $S(z)$ м.p. $S(\delta)$.

В [5] показано, что для любого $z$ из нижней полуплоскости операторы $L_{z}=$ $\left.B^{* 2}\right|_{D\left(L_{z}\right)}, D\left(L_{z}\right)=P\left(\widetilde{L}-z^{2} I\right)^{-1} \mathfrak{H}(P-$ ортопроектор в $\widetilde{\mathfrak{H}}$ на $\mathfrak{H})$, корректно определены в $\mathfrak{H}$. При этом $z^{2} \in \rho\left(L_{z}\right)$ и

$$
P\left(\widetilde{L}-z^{2} I\right)^{-1} f=\left(L_{z}-z^{2} I\right)^{-1} f \quad \forall f \in \mathfrak{H} .
$$

Множество операторов $\left\{L_{z} \mid \operatorname{Im} z<0\right\}$ будем называть множеством образов возмущенного оператора $\widetilde{L}$. Для описания этого множества используем понятие позитивного пространства граничных значений (ПГЗ) [6]. Положим $\mathcal{H}=$ $\operatorname{ker}\left(B^{* 2}+I\right)$. Тогда $D\left(B^{* 2}\right)=D\left(L_{\mu}\right) \dot{+} \mathcal{H}$, где $L_{\mu}=B^{*} B$. Тройка $\left(\mathcal{H}, \Gamma_{1}, \Gamma_{2}\right)$, где операторы $\Gamma_{i}: D\left(B^{* 2}\right) \rightarrow \mathcal{H}$ определяются формулами $\Gamma_{1}(u+h)=P_{\mathcal{H}}\left(L_{\mu}+I\right) u$ и $\Gamma_{2}(u+h)=h$ (здесь $u \in D\left(L_{\mu}\right), h \in \mathcal{H}$ и $P_{\mathcal{H}}$ - ортопроектор в $\mathfrak{H}$ на подпространство $\mathcal{H})$, является позитивным ПГЗ оператора $B^{2}$, построенным по расширению Фридрихса $L_{\mu}$. В этом ПГЗ область определения оператора $L_{z}$ представима в виде

$$
D\left(L_{z}\right)=\left\{f=u+h\left(f \in D\left(B^{* 2}\right)\right) \mid C_{z} \Gamma_{1} f=\Gamma_{2} f\right\},
$$

где ограниченный оператор $C_{z}$ однозначно определяется в $\mathcal{H}$. Отметим, что вспомогательное пространство $N$ из определения м.р. и $\mathcal{H}$ имеют одинаковую размерность и, следовательно, изоморфны. Поэтому можно считать, что $N=\mathcal{H}$. 
Доказательство следующей теоремы проводится с помощью абстрактных методов теории расширений и базируется на известном результате В. М. Адамяна и Д. 3. Арова [7] о связи между м.р. и характеристической функцией сжатия.

ТЕорема 1. Аналитическое продолжение в нижнюю полуплоскость м.р. $S(\delta)$ имеет вид

$$
S(z)=I-4 i z C_{z}\left(I-2(1-i z) C_{z}\right)^{-1},
$$

где операторь $C_{z}$ определяют множество образов оператора $\widetilde{L}$ в (3). При этом точка $1 / 2(1-i z)$ принадлежит резольвентному множеству оператоpa $C_{z}$.

3. Обратная задача. Пусть функция $S(\delta)$ - граничное значение в смысле сильной сходимости аналитической в нижней полуплоскости функции $S(z)$, значения которой являются сжимающими операторами в $N$, и пусть ненулевое дефектное число оператора $B$ совпадает с размерностью пространства $N$. Рассмотрим следующие задачи:

1. Когда функция $S(z)$ определяет м.р. для возмущенного уравнения (1)?

2. Возможно ли по известной м.р. $S(\delta)$ определить соответствующее возмущенное расширение $\widetilde{L}$ оператора $B^{2}$ ?

ТЕОРема 2. Функция $S(\delta)$ является м.р. для возмущенного уравнения (1) тогда и только тогда, когда ее аналитическое продолжение в нижнюю полуплоскость $S(z)$ удовлетворяет условию $S^{*}(z)=S(-\bar{z})$ (для любого $z$, $\operatorname{Im} z<0)$. При этом функиия $S(\delta)$ будет м.р. для уравнения (1) с 0-возмущеннылм оператором $\widetilde{L}$ в правой части тогда и только тогда, когда существует точка $z, \operatorname{Im} z<0, \operatorname{Re} z \neq 0$, для которой справедливо равенство $(\operatorname{Re} z)\left(I-S^{*}(z) S(z)\right)=2(\operatorname{Im} z) \operatorname{Im} S(z)$.

Возмущенный оператор $\widetilde{L}$ называется минимальныл, если ни одно из нетривиальных подпространств пространства $\widetilde{\mathfrak{H}} \ominus \mathfrak{H}$ не приводит оператор $\widetilde{L}$.

Минимальные возмущенные расширения $\widetilde{L}_{1}$ и $\widetilde{L}_{2}$ оператора $B^{2}$, действующие в пространствах $\widetilde{\mathfrak{H}}_{1}$ и $\widetilde{\mathfrak{H}}_{2}$ соответственно, называются эквивалентныли, если существует изометрическое отображение $X$ пространства $\widetilde{\mathfrak{H}}_{1}$ на $\widetilde{\mathfrak{H}}_{2}$, такое, что $\widetilde{L}_{2}=X \widetilde{L}_{1} X^{-1}$ и $X f=f$ для $f \in \mathfrak{H}$.

Теорема 3. Аналитическое продолжение $S(z)$ м.p. $S(\delta)$ однозначно, с точностью до эквивалентности, определяет минимальное возмущенное расширение $\widetilde{L}_{\text {в }}(1)$.

Доказательства теорем 2 и 3 основаны на следующем наблюдении: в силу равенств (2)-(4) по аналитическому продолжению $S(z)$ можно определить обобщенную резольвенту $R_{\mu}=\left(L_{z}-\mu I\right)^{-1}\left(\mu=z^{2}\right)$ симметрического оператора $B^{2}$. Используя этот факт и результаты М. А. Наймарка (теорема 8 в [8]) и А. В. Штрауса (теорема 7 в [9]), мы завершаем доказательства этих теорем.

СлеДСтВИЕ 1. М.p. $S(\delta)$ является унитарной константой тогда и только тогда, когда оператор $\widetilde{L}_{\text {в }}$ (1) является невозмущенныл оператором.

ЗАмечАния. 1. Доказательство теоремы 3 не дает конструктивного алгоритма восстановления возмущенного оператора $\widetilde{L}$ по его множеству образов. Эта проблема остается открытой. 
2. Используя [10], теоремы 1-3 можно обобщить на случай, когда возмущенный оператор имеет конечный дискретный спектр на отрицательной полуоси.

\section{ЛИТЕРАТУРА}

1. Kuzhel A. V., Kuzhel S. A. Regular extensions of Hermitian operators. VSP, Utrecht (1998). 2. Кужель C. А. Укр. матем. ж., 50, № 12, 1615-1629 (1998). 3. Кужель C. A. Функц. анализ и его прил., 30, вып. 1, 70-73 (1996). 4. Лакс П., Филлипс Р. Теория рассеяния. Мир, М. (1971). 5. Штраус А. В. Изв. АН СССР, сер. матем., 29, № 6, 1389-1416 (1965). 6. Горбачук В. И., Горбачук М. Л. Граничные задачи для дифференциально-операторных уравнений. Наукова думка, Киев (1984). 7. $A \partial a-$ мян В. М., Аров Д. З. ДАН СССР, 160, № 1, 9-12 (1965). 8. Наймарк М. А. Изв. АН СССР, сер. матем., 4, № 3, 277-309 (1940). 9. Шmpayc А. В. Изв. АН СССР, сер. матем., 18, № 1, 51-86 (1954). 10. Кужель С. А. Матем. сб., 187, № 10, 87-108 (1996).

Институт Математики НАН Украины e-mail: kuzhel@imath.kiev.ua

Поступило в редакцию 18 декабря 1998 г.

\section{УДК 517.98}

\section{Рекуррентные формулы для $A_{n}$ - и $B_{n}$-решений уравнения WDVV*}

(C) 2000. C. M. НАтАнзОН

1. В начале $90-$ х годов в работах $[5,8]$ была построена система дифференциальных уравнений (называемая сейчас WDVV), описывающая древесное приближение 2-мерных топологических теорий поля при нулевых константах гравитационного спаривания. Дубровин [3] обнаружил, что решения этой системы описывают замечательную дифференциально-геометрическую структуру Фробениуса-Дубровина, связывающую самые, казалось бы, далекие разделы математики: алгебраическую геометрию, дискретные группы, квантовые когомологии, теорию особенностей, интегрируемые системы и др.

Простейшими нетривиальными решениями WDVV являются так называемые $A_{n}-u B_{n}$-потенииальь [5-7, 9], описывающие, как оказалось впоследствии, метрику K. Саито на пространстве версальных деформаций особенностей $A_{n}$ и $B_{n}$.

$A_{n-1}$-потенциалы $F_{A}\left(x_{1}, \ldots, x_{n-1}\right)$ и $B_{n-1}$-потенциалы $F_{B}\left(x_{1}, \ldots, x_{n-1}\right)$ определяются как функции, удовлетворяющие системе уравнений

$$
\begin{gathered}
\sum_{\gamma=1}^{n-1} \frac{\partial^{3} F}{\partial x_{\alpha} \partial x_{\beta} \partial x_{\gamma}} \frac{\partial^{3} F}{\partial x_{n-\gamma} \partial x_{\eta} \partial x_{\xi}}=\sum_{\gamma=1}^{n-1} \frac{\partial^{3} F}{\partial x_{\xi} \partial x_{\beta} \partial x_{\gamma}} \frac{\partial^{3} F}{\partial x_{n-\gamma} \partial x_{\eta} \partial x_{\alpha}}, \\
\frac{\partial^{3} F}{\partial x_{1} \partial x_{\alpha} \partial x_{\beta}}=\delta_{\alpha+\beta, n}, \quad \sum_{j=1}^{n-1} d_{i} \frac{\partial F}{\partial x_{i}}=d F,
\end{gathered}
$$

*Работа выполнена при частичной финансовой поддержке грантов РФФИ (98-01-00612) и INTAS. 